\title{
GROUPS PRESENTED BY CERTAIN CLASSES OF FINITE LENGTH-REDUCING STRING-REWRITING SYSTEMS
}

\author{
Klaus Madlener \\ Friedrich otto \\ Fachbereich Informatik, Universität Kaiserslautern \\ 6750 Kaiserslautern, Fed. Rep. Germany

\begin{abstract}
The second author is currently visiting at Department of Computer Science, State University of New York

Albany, N.Y. 12222 , U.S.A.
\end{abstract}

\section{Introduction}

A string-rewriting system $R$ on alphabet $\Sigma$ defines a monoid $M_{R^{\prime}}$ which is the factor monoid of the free monoid $\Sigma *$ generated by $\Sigma$ modulo the Thue congruence $\stackrel{*}{\longleftrightarrow}$ generated by $R$. One aspect of the various classes of string-rewriting systems considered in the literature is their descriptive power: which monoids can be defined by which classes of string-rewriting systems. To capture the descriptive power of string-rewriting systems one would like to establish a one-to-one correspondence between the various syntactic restrictions of string-rewriting systems and certain algebraic properties of the monoids presented.

So far only a few results of this flavor have been obtained most of them dealing with groups rather than with monoids in general. Here we shall also restrict our attention to the case of groups. So we ask which classes of groups can be presented by which classes of string-rewriting systems.

In this paper we give an overview over the results concerning this question that have been obtained so far, including some new ones. Specifically, we are interested in the following classes of finite length-reducing string-rewriting systems:

- $C_{\text {Sp' }}$ the class of finite special systems $R$ that are confluent, - $C_{S p, 1}$, the class of finite special systems $R$ that are confluent on $[1]_{R}$ and that provide an inverse of length 1 for each of the generators, 
- $C_{2 m}$ the class of finite 2-monadic systems $R$ that are confluent,

- $C_{m}$, the class of finite monadic systems $R$ that are confluent

- $\mathrm{C}_{\mathrm{m}, \mathrm{I}}$ the class of finite monadic systems $\mathrm{R}$ that are confluent on $[1]_{R}$ and that provide inverses of length 1 for the generators,

- $C_{1 r}$ the class of finite length-reducing systems $\mathrm{R}$ that are confluent, and

- $\mathrm{C}_{1 \mathrm{r}, 1}$, the class of finite length-reducing systems $\mathrm{R}$ that are confluent on $[1]_{R}$ and that provide inverses of length 1 . the generators.

Here $I$ denotes the empty word, and $[w]_{R}$ denotes the congruence class of $w \in \Sigma *$ modulo the congruence $\stackrel{*}{\longleftrightarrow} R$. A string-rewriting system $R$ on $\Sigma$ is called length-reducing, if $|I|>|r|$ for each rule $(I, Y) \in R$, where $1.1: \Sigma * \rightarrow N$ denotes the usual length function. It is called monadic, if it is length-reducing and $|r| \leqslant 1$ for each rule $(1, x) \in R$, and it is called 2 -monadic, if it is monadic and $111 \leqslant 2$ for each rule $(1, r) \in R$. Finally, $R$ is called special, if it is lengthreducing and the right-hand side $r$ of each rule $(1, r) \in R$ is the empty word 1 . A string-rewriting system $R$ on $\Sigma$ is confluent, if each of its congruence classes contains a unique irreducible word, which can then be taken as the normal form of its class. Since the process of reduction in a finite length-reducing system can be performed in linear time, the word problem for each finite length-reducing and confluent system is decidable in Iinear time [3]. Finally, a string-rewriting system $R$ on $\Sigma$ is said to be confluent on $[1]{ }_{R^{\prime}}$ if $w_{\mathrm{R}}^{*} \stackrel{*}{\rightarrow}_{\mathrm{f}} I$ for each word $w \in[1]_{\mathrm{R}}$. Since we are dealing with groups, confluence on $[1]_{R}$ is sufficient to guarantee that the word problem of a finite length-reducing $R$ is decidable in linear time. For more details on the concepts introduced so far, see for example Book's excellent overview paper [4].

The following overview summarizes the results that we shall present here. 


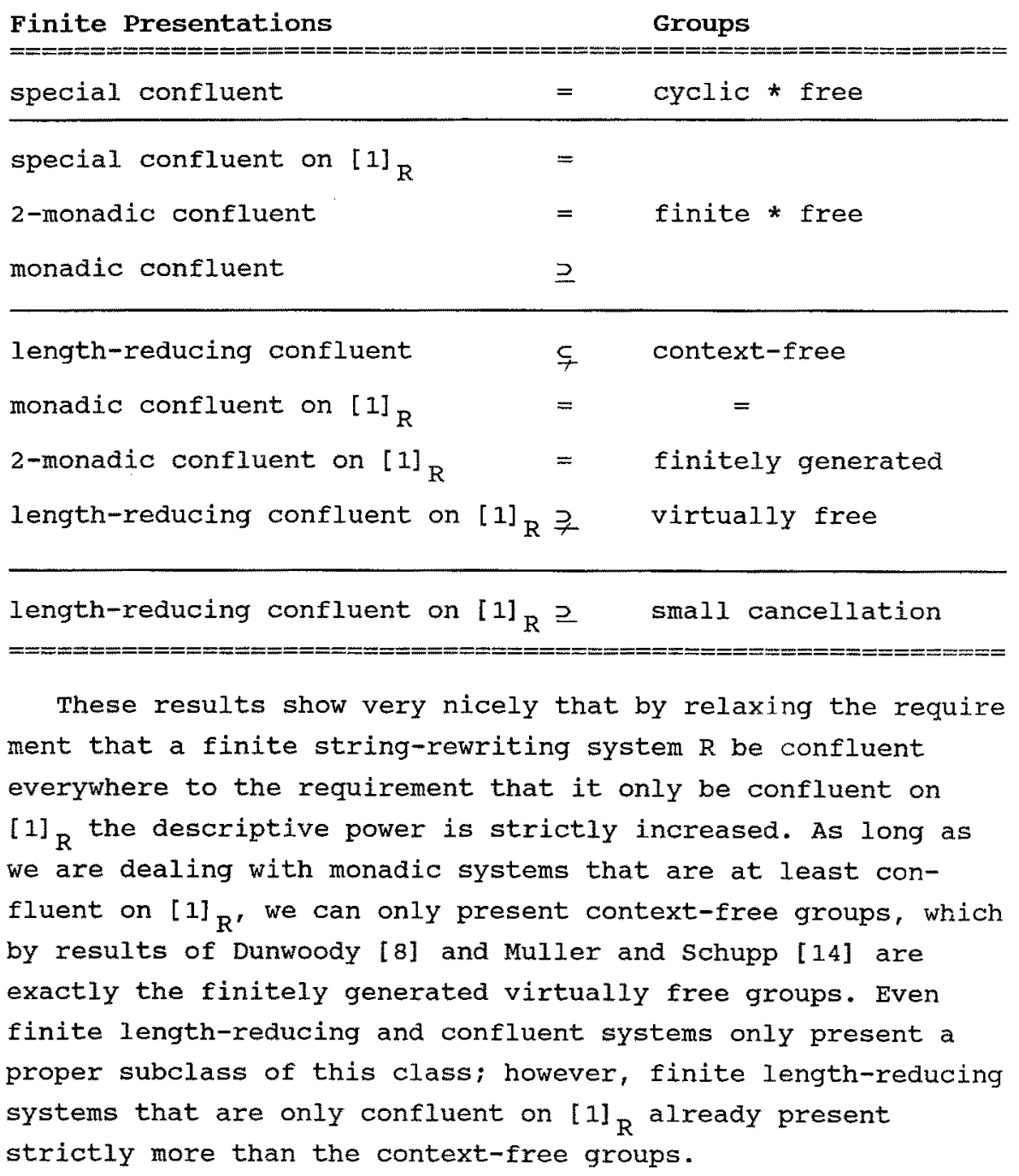

\section{Special and monadic string-rewriting systems}

The first characterization theorem is due to cochet.

Theorem 2.1 [6]. A group $G$ has a presentation of the form $(\Sigma ; R)$, where $R$ is a finite special confluent system on $\Sigma$, if and only if $G$ is the free product of finitely many cyclic groups.

Here the factors of $G$ may be finite or infinite cyclic 
groups. This result takes care of the class $\mathrm{C}_{\mathrm{sp}}$. It is easily seen that every group $G$ that is the free product of finitely many cyclic groups can be presented by a finite special string rewriting system $R$ on alphabet $\Sigma$ such that $R$ is confluent on $[1]_{R}$ and such that each letter $a \in \Sigma$ has an inverse $b \in \Sigma$. Hence, the descriptive power of the class $\mathrm{C}_{\mathrm{sp}, 1}$ (with respect to the presentation of groups) is at least as large as the descriptive power of the class $c_{s p}$. In fact, it is even larger, as shown by the following lemma.

Lemma 2.2. Let $G$ be a free product of a free group of finite rank and a finite number of finite groups. Then $G$ has a presentation $(\Sigma ; R)$ for some finite string-rewriting system $R$ from the class $\mathrm{C}_{\text {sp, }}$.

Proof. If $G_{1}$ and $G_{2}$ have presentations of this form, say $\left(\Sigma_{1} ; R_{1}\right)$ and $\left(\Sigma_{2} ; R_{2}\right)$ with $\Sigma_{1} \cap \Sigma_{2}=\not$, then $\left(\Sigma_{1} u \Sigma_{2} ; R_{1} u R_{2}\right)$ is a presentation of this form for the free product $G_{1} * G_{2}$. If $G$ is a free group, then the standard presentation $(\bar{\Sigma} \cup \bar{\Sigma} ; \mathrm{a} \bar{a} \rightarrow 1$, $\bar{a} a \rightarrow 1 \mid a \in \Sigma\})$ is of this form. Thus, it only remains to consider the case that $G$ is an arbitrary finite group.

So let $G=\left\{1_{G}, g_{1}, \ldots, g_{n-1}\right\}$ be a finite group, where $1_{G}$ denotes the identity of $G$ and - denotes the operation of $G$. Let $\Sigma=\left\{a_{1}, \ldots, a_{n-1}\right\}$, and let $f: \Sigma * \rightarrow G$ denote the monoidhomomorphism induced by $a_{i} \rightarrow g_{i}(i=1,2, \ldots, n-1)$. Further, let $R=\left\{(w, I)\left|W \in I_{*}, 1<\right| w \mid \leq n, f(w)={ }_{G} I_{G}\right\}$, where $=_{G}$ denotes the equality in $G$.

claim 1. Let $w \in I *,|w|>n$, such that $f(w)={ }_{G} 1_{G}$. Then $w$ can be factored as $w=x y z$ with $|Y| \leq n$ such that $f(y)={ }_{G}{ }^{1} G$. Proof. Let $w=a_{1} a_{2} \ldots a_{m}, m>n$, with $a_{1}, a_{2}, \ldots, a_{m} \in \Sigma$, and let $e_{i_{j}}=f\left(a_{1} a_{2} \ldots a_{j}\right)$ for $j=1,2, \ldots, m$. Since $m>n$, there exist indices $1 \leq j<k \leq j+n$ such that $e_{i_{j}}=e_{i_{k}}$. Thus, $f\left(a_{1} \ldots a_{j}\right)={ }_{G} e_{i_{j}}=e_{i_{k}}={ }_{G} f\left(a_{1} \ldots a_{j} a_{j+1} \ldots a_{k}\right)$ implying that $f\left(a_{j+1} \cdots a_{k}\right)={ }_{G}{ }_{G}$, i.e., $y=a_{j+1} \ldots a_{k}$ satisfies the requirements, a

In particular, this means that $W^{*} \stackrel{\rightarrow}{R} 1$ for all $w \in \Sigma *$ satisfying $f(W)={ }_{G}{ }^{1} \cdot$. Further $(\Sigma ; R)$ is a presentation of $G$ as shown by the following claim. 
claim 2. $\forall u, v \in \Sigma *: u \stackrel{*}{\longleftrightarrow}{ }_{R} v$ if and only if $f(u)={ }_{G} f(v)$. Proof. Let $u, v \in \Sigma^{*}$. If $u \stackrel{*}{\longleftrightarrow} v$, then $u \longleftrightarrow u_{R} u_{1} \longleftrightarrow R_{R} \cdots$ $\longleftrightarrow_{R} u_{r}=v$. Since $(w, 1) \in R$ implies $f(w)={ }_{G} 1_{G}=f(1)$, it can be verified easily by induction on $r$ that $f(u)={ }_{G} f(v)$. on the other hand, if $f(u)={ }_{G} f(v)$, then $f(u)(f(v))^{-1}={ }_{G}{ }^{1}{ }_{G}$ Since $G$ is a group, there exists an element $g_{i}^{-1} \in\left\{g_{1}, g_{2}, \ldots\right.$, $g_{n-1}$ ) for each element $g_{i}$, and hence a function $(.)^{-1}: \Sigma * \rightarrow \Sigma *$ can be defined. Now $f\left(v^{-1}\right)={ }_{G}(f(v))^{-1}$, and so $f(u)(f(v))^{-1}={ }_{G}$

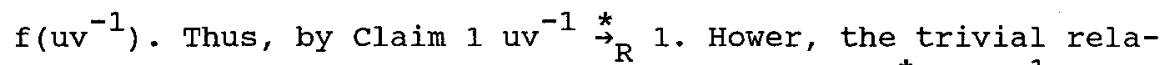
tions $(\bar{a} a, l)$ are in $R$ for all a $\in \Sigma$, and so $u \stackrel{{ }_{*}}{\longleftrightarrow} u v^{-1} v$ $\stackrel{*}{\longleftrightarrow} \mathrm{R} \cdot \mathrm{v}$

Since $R \in C_{s p, 1}$, this completes the proof of Lemma 2.2. If a group $G$ has a presentation of the form $(\Sigma, R)$ with $R \in C_{s p, 1}$, then because of the inverses of length 1 for the generators $a \in \Sigma,(\Sigma ; R)$ is in fact a group presentation in the sense of [13]. Further, the set $W(R)=\{W \in \Sigma * 1 W \stackrel{*}{\longleftrightarrow} 1$, but no proper factor $u$ of $w$ satisfies $\left.u \stackrel{*}{\longleftrightarrow_{R}} 1\right\}$ is a subset of the set of left-hand sides of rules of $R$, and hence it is finite. Thus, the following lemma applies.

Lemma $2.3[10]$. Let $(\Sigma ; R)$ be a group presentation such that the set $W(R)$ is finite. Then the group $G$ presented by $(\Sigma ; R)$ is the free product of a finitely generated free group and finitely many finite groups.

Hence, we have the following characterization theorem.

Theorem 2.4. A group $G$ has a presentation of the form $(\Sigma ; R)$, where $R$ is a finite special string-rewriting system on $\Sigma$ such that $R$ is confluent on $[1]_{R}$ and each generator $a \in \Sigma$ has an inverse $b \in \Sigma$, if and only if $G$ is the free product of a free group of finite rank and a finite number of finite groups.

These are exactly those groups that have a presentation with a simple reduced word problem [10]. Another characterization of this class of groups has been obtained by Avenhaus, Madlener, and otto. 
Theorem $2.5[2]$. A group $G$ has a presentation of the form $(\Sigma ; R)$, where $R$ is a finite 2 -monadic and confluent string-rewriting system on $\Sigma$, if and only if $G$ is the free product of a free group of finite rank and a finite number of finite groups.

It has been conjectured [9] that when we take presentations involving finite monadic confluent systems, we will still have the same class of groups. Up to now this conjecture is still open. On the other hand, whenever $R$ is a finite monadic confluent string-rewriting system on $\Sigma$, then $[1]_{R}$ is a contextfree language [3]. Thus, if the monoid $M_{R}$ presented by $(\Sigma ; R)$ is a group, then it is a context-free group. Muller and Schupp have characterized the class of context-free groups as follows.

Theorem 2.6 [14]. A finitely generated group $G$ is context-free if and only if it is virtually free.

A group $G$ is called virtually free, if it contains a free subgroup of finite index. Actually, Muller and Schupp showed that a finitely generated group $G$ is virtually free if and only if it is context-free and accessible. However, since every finitely generated context-free group is finitely presented [1], and since every finitely presented group is accessible [8], the condition of accessibility can be dropped from the Muller-Schupp Theorem. Thus, we know at least that evey group that is presented by a finite monadic confluent string-rewriting system is virtually free.

When $R$ is a finite monadic string-rewriting system that is confluent on $[1]{ }^{\prime}$, then $[1]{ }_{R}$ is still a context-free language. Thus, whenever a group $G$ is presented by a system of this form, then $G$ is a virtually free group. On the other hand, each finitely generated virtually free group can be presented by a quadratic NTS g-grammar, and hence the group language of $G$ is an NTS language [16]. Since a quadratic NTS g-grammar yields a finite 2 -monadic string-rewriting system $R$ on alphabet $\Sigma$ such that $R$ is confluent on $[1]_{R}$ and such that each generator $a \in \Sigma$ has an inverse $b \in \Sigma$, this shows the following.

Theorem 2.7 [16]. A group $G$ has a presentation $(\Sigma ; R)$, where $R$ is a finite monadic string-rewriting system on $\Sigma$ that is confluent on $[1]_{R}$ and that provided inverses of length 1 for all the generators $a \in \Sigma$, if and only if $G$ is a finitely generated virtually free group. 


\section{Length-reducing string-rewriting systems}

Let $\mathrm{R}$ be a finite length-reducing and confluent string-rewriting system on $\Sigma$. Then for each word $w \in \Sigma *$, the congruence class [w] ${ }_{R}$ is a context-sensitive language [3], but in general ${ }^{[W]}{ }_{R}$ is not context-free [15] . However, if the monoid $M_{R}$ presented by $(\Sigma ; R)$ is a group, then the situation is different.

Theorem 3.1. Let $R$ be a finite length-reducing and confluent string-rewriting system on $\Sigma$, and let $L \subset I R R(R)$ be a regular set of irreducible words. If the monoid $M_{R}$ is a group, then the $\operatorname{set}[L]_{R}:=\left\{u \in \Sigma *_{\mid} \exists W \in L: u \stackrel{{ }^{*}}{\longleftrightarrow} w\right\}$ is a deterministic context-free language.

Proof, Since $M_{R}$ is a group, there exists a word $u_{a} \in \Sigma^{*}$ such that $a_{a} \stackrel{*}{\rightarrow}_{R} 1$ and $u_{a} a \stackrel{*}{\rightarrow}_{R} 1$ for each letter $a \in \Sigma$. Let $\lambda:=\max$ $\left\{11 \| \exists r \in \Sigma^{*}:(1, r) \in \mathbb{R}\right\}$, and $\mu:=\left(\max \left\{\left|u_{a}\right| \mid a \in \Sigma\right\}+1\right)(\lambda-1)$. Now for $u \in \operatorname{IRR}(R)$, if $|u| \geq \mu$ and $u a \stackrel{*}{\rightarrow} \vee v \in \operatorname{IRR}(R)$ for some $a \in \Sigma$, then $u \stackrel{*}{R}_{R}^{*} u_{a u_{a}} \stackrel{*}{R}_{R} v u_{a}$ implying that $v_{a} \stackrel{*}{\rightarrow}_{R} u$. Thus, $|v| \leq|u|+1$ and $|u| \leq|v|+\left|u_{a}\right|$, i.e., $0 \leqslant|u a|-|v| \leqslant \mid u a^{1+1}$. since $R$ is length-reducing, this means that ua $\stackrel{1}{A}_{R} v$ for some $i \leqslant\left|u_{a}\right|+1$, and since $u$ is irreducible, $u=u_{1} u_{2},\left|u_{2}\right| \leqslant$ $\left(\left|u_{1}\right|+1\right)(\lambda-1) \leq \mu$ such that $u_{2} a^{a} \stackrel{*}{\rightarrow}_{R} v_{2}$ and $v=u_{1} v_{2}$. Thus, in order to reduce ua only the suffix $u_{2}$ of $u$ of length $\mu$ must be considered.

Now we construct a deterministic pushdown automaton (dpda) C that will accept the language $[L]_{R}=\left\{u \in \Sigma * \mid \exists w \in L: u \stackrel{*}{\rightarrow}_{R} w\right\}$ o

First we construct a dpda $A$ as follows. As input alphabet we take $\Sigma$, and as stack alphabet we choose $\Sigma \cup\{\#\}$, where \# serves as the start symbol marking the bottom of the pushdown store. A can store a word of length $\leqslant \mu$ in its finite control, i.e., the set of finite states is $Q=\left(U_{i}^{\mu}=0 \Sigma^{i}\right) \cap \operatorname{IRR}(R)$. Finally, the transition function $\delta: \operatorname{Qx}(\Sigma \cup\{\#\}) \times(\Sigma \cup\{1\}) \rightarrow$ $Q \times(\Sigma \cup\{\#\})$ * is defined as follows:

$$
\begin{aligned}
& \delta(w, \#, a):= \begin{cases}(u, \#) & \text { if } w a \stackrel{*}{\rightarrow}_{R} u \in \operatorname{IRR}(R) \text { and }|u| \leq \mu, \\
(v a, \# b) & \text { if } w=b v,|w|=\mu, \text { and wa } \in \operatorname{IRR}(R),\end{cases} \\
& \delta(w, b, 1):=(b w, 1) \quad \text { if }|w|<\mu,
\end{aligned}
$$


$\delta(w, b, a):= \begin{cases}(v a, b c) & \text { if } w=c v,|w|=\mu, \text { and } w a \in \operatorname{IRR}(R), \\ (u, b) & \text { if }|w|=\mu, \text { wa } \stackrel{*}{\rightarrow} R \in \operatorname{IRR}(R) \text { and } \\ \text { iu } \leq \mu .\end{cases}$

Starting with the configuration $(1, w, \#)$, where 1 is the initial state, $W$ is the input, and \# is the stack contents, A finally reaches a configuration of the form $(V, I, H u)$ such that $\mathrm{W} \stackrel{*}{\rightarrow} \mathrm{R}$ uv and $u v \in \operatorname{IRR}(\mathrm{R})$. Thus, $\mathrm{W} \in[\mathrm{L}]_{\mathrm{R}}$ if and only if $u v \in L$.

Since $I$ is regular, there exists a deterministic finite state acceptor B for I. Now $A$ and $B$ can be conbined such that for each configuration $(u, w, \# v)$ of $A, B$ is in its state ${ }_{B}(v u)$ This gives a dpaa $C$ for $[L]_{R}$.

In particular, each congruence class $[\mathrm{W}]_{R}$ of a finite length-reducing confluent string-rewriting system $R$ presenting a group is context-free, which implies the following result.

Corollary 3.2. Let $G$ be a group that can be presented by a finite length-reducing and confluent string-rewriting system. Then $G$ is a context-free group.

This result was independently obtained by $V$. Diekert [7], who also proved the following.

Theorem $3.3[7]$. Let $G$ be an infinite group that has an abelian subgroup of finite index. Then $G$ has a presentation by some finite length-reducing and confluent string-rewriting system if and only if $G$ is isomorphic to $z$ or to the free product $\mathrm{z}_{2} * \mathrm{z}_{2}$.

Concerning commuting elements in groups presented by finite length-reducing and confluent systems the following result can be shown.

Lemma $3.4[12]$. Let $\mathrm{R}$ be a finite length-reducing and confluent string-rewriting system on $\Sigma$ such that the monoid $M_{R}$ is a group, and let $u \in \Sigma *$ be a word of infinite order. Then, for all $\mathrm{V} \in \Sigma^{*}$, the following two statements are equivalent:

(i) $\mathrm{u}$ and $\mathrm{V}$ commute modulo $\mathrm{R}$;

(ii) the subgroup of $M_{R}$ generated by $\{u, v\}$ is cyclic. 
As a consequence of this lemma we obtain the following results.

Theorem 3.5 [12]. Let $G$ be a group that can be presented by $a$ finite length-reducing and confluent string-rewriting system. Then for each element $g$ of $G$, if $g$ has infinite order, then the centralizer $\mathrm{C}_{\mathrm{G}}(\mathrm{g})$ of $\mathrm{g}$ in $\mathrm{G}$ is isomorphic to $\mathrm{z}$.

Corollary 3.6. Let $G$ be a group that can be presented by a finite length-reducing and confluent string-rewriting system. (a) Every abelian subgroup of $G$ that contains an element of infinite order is isomorphic to $z$.

(b) Every finitely generated abelian subgroup of $G$ is either finite or isomoprhic to $\mathrm{z}$.

(c) If the center of $G$ is non-trivial, then $G$ is either finite or isomorphic to $\mathrm{z}$.

(d) If $G$ contains a non-trivial normal subgroup that is finite then $G$ itself is finite.

This corollary is very important in that it provides ways of proving that a certain group cannot be presented by a finite length-reducing and confluent string-rewriting system. For example, consider $\mathrm{G}=\mathrm{F}_{2} \mathrm{XZ}_{2}$, i.e., $\mathrm{G}$ is the direct product of the free group $\mathrm{F}_{2}$ of rank 2 and the cyclic group $\mathrm{z}_{2}$ of order 2. Then $G$ is an infinite context-free group, but $z_{2}$ is a finite nomal subgroup of $G$. Hence, $G$ cannot be presented by a finite length-reducing and confluent system. Thus, we have the following.

Corollary 3.7. The class of groups that can be presented by finite length-reducing and confluent string-rewriting systems is a proper subclass of the class of finitely generated virtually free groups.

By definition a virtually free group $G$ contains a free subgroup $H$ of finite index. If $H \cong z$, then $G$ has a linear growth function, otherwise, $G$ has an exponential growth function. Now if $H \cong Z$, then $H$ is an abelian subgroup of finite index of $G$. Thus, if $G$ has a presentation by some finite length-reducing and confluent string-rewriting system, then $G$ is isomorphic to $z$ or to $z_{2} * z_{2}$. 
corollary 3.8. Let $G$ be an infinite group that is presented by a finite length-reducing and confluent string-rewriting system Then either $G$ is isomorphic to $z$, or to $z_{2} * z_{2}$, in which cases $G$ has a Iinear growth function, of $G$ has an exponential growth function.

Since a finitely generated nilpotent group has a polynomial growth function $[17], z$ is the only infinite nilpotent group that can be presented by a finite length-reducing and confluent string-rewriting system.

Pinally, we turn to string-rewriting systems $R$ that are length-reducing, confluent on $[1]_{R^{\prime}}$ and that provide inverses of length 1 for the generators.

From the result in [16], which says that each context-free group can be presented by a quadratic NTS g-grammar, it follows that each context-free group has a presentation of the form $(\Sigma ; R)$, where $R$ is a finite monadic string-rewriting system that is confluent on $[l]_{R}$ and that provides inverses of length 1 for the generators $a \in \Sigma$. Thus, we do in particular have the following weaker result.

Theorem 3.9. Each finitely generated virtually free group $G$ has a presentation of the form $(\Sigma ; R)$, where $R$ is a finite length-reducing string-rewriting system on $\Sigma$ that is confluent on $[1]_{R}$ and that provides inverses of length 1 for the generators $a \in \Sigma$.

However, this result can be derived immediately from the fact that a finitely generated virtually free group $G$ is context-free using an idea of [1].

Proof of Theorem 3.9. Let $\langle r ; L\rangle$ be a finite group-presentation of $G$, where $L \subset(r \cup r)$. Since $G$ is context-free, [1] $L$ is a context-free language over $\Sigma:=\Gamma \cup \Gamma^{-}$. Let $n_{1}:=\max (\{|W|\}$ $w \in I\} \cup\{2\})$, and let $n_{2}$ be the constant of the pumping lemma belonging to $[1] \mathrm{L}$. Finally, let $\mathrm{n}:=\max \left\{\mathrm{n}_{1}, \mathrm{n}_{2}\right\}$, and $\mathrm{R}:=$ $\left\{(u, 1) \mid u==_{G} 1\right.$ and $\left.|u| \leqslant n\right\} u\left\{(x y z, y)\left|x y z={ }_{G} y,\right| x y z \mid \leqslant n\right.$, and $x z \neq 1\}$.

Then $(w, 1) \in R$ for all words $w \in L$, and $(\bar{a} a, 1),(a \bar{a}, 1) \in R$ for all $a \in I$, i.e., $G \cong \mathbb{M}_{R}=\Sigma * / \stackrel{*}{\longleftrightarrow}_{R^{*}}$ obviously, $R$ is a finite length-reducing string-rewriting system on $\Sigma$ providing inverses of length 1 for the generators. 
Now let $w \in[1]_{L}=[1]_{R}$ If $|w| \leq n$, then $(w, 1) \in R$, i.e., $\mathrm{w} \rightarrow_{\mathrm{R}} 1$. If $|\mathrm{w}|>\mathrm{n}$, then $\mathrm{w}=$ uxyzv, where $|x y z| \leq \mathrm{n}, \mathrm{xz} \neq 1$, and $\left\{u^{k} z^{k} v \mid k \geq 0\right\} \subset[1]_{R}$ by the pumping lemma for contextfree languages. In particular, uyv ${ }_{G}$ uxyzv implying $x y z={ }_{G} y$. Thus, $(x y z, y) \in R$, and so $w=u x y z v \rightarrow_{R}$ uyv. Inductively, we

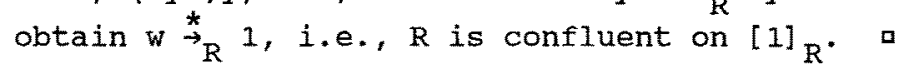

Dehn's algorithm [11] for solving the word problem actually amounts to applying a finite length-reducing string-rewriting system $R$ that is confluent on $[1]{ }_{R}$. Thus, all classical small cancellation groups have presentations by rewriting systems $R$ from the class $G_{1 r, 1}$. This fact has been first observed by Buecken [5] who investigates the class of small cancellation groups from the standpoint of rewriting systems. On the other hand, the Fuchsian group $G_{2}=\langle a, b, c, d$; $a b \bar{a} \bar{b}=d c \bar{d} \bar{c}\rangle$ satisfies the small cancellation condition $C^{\prime}(1 / 7)$, i.e., $G_{2}$ has a presentation by some system $R$ from $C_{1 r, 1} \cdot G_{2}$ is torsionfree, since it is the free product of the free group $F_{2}$ with itself with amalgamated cyclic subgroups, but $G_{2}$ is not a free group. Hence, $G_{2}$ is not a context-free group, and so it does not have a presentation by some finite length-reducing and confluent string-rewriting system. This gives the following.

Theorem 3.10. The class of finitely generated virtually free groups is a proper subclass of the class of groups that can be presented by finite length-reducing string-rewriting systems $R$ that are confluent on $[1]_{R}$ and that provide inverses of length 1 for the generators.

It is conjectured that the classes $C_{2 m}, C_{m}$ and $C_{1 r}$ are all equal to the class of free products of a free group of finite rank and a finite number of finite groups. The proof of this fact would give a complete algebraic characterization of groups presented by length-reducing systems. 


\section{References}

1. A.W. Anissimov, F.D. Seifert; Zur algebraischen charakteristik der durch kontext-freie sprachen definierten Gruppen; Elektr. Informationsverarbeitung und Kybernetik EIK 11(1975), 695-702.

2. J. Avenhaus, K. Madlener, F. Otto; Groups presented by finite two-monadic Church-Rosser Thue systems; Trans. Amer. Math. Soc. 297(1986), 427-443.

3. R.V. Book; Confluent and other types of Thue systems; Journal Assoc. Comput. Mach. 29(1982), 171-182.

4. R.V. Book; Thue systems as rewriting systems, in: J.P. Jouannaud (ed.), Rewriting Techniques and Applications; Lect. Notes Comp. Sci. $202(1985), 63-94$.

5. H.Buecken; Reduction systems and small cancellation theory; Proc. 4th Workshop on Automated Deduction, $1979,53-59$.

6. Y. Cochet; Church-Rosser congruences on free semigroups; Coll. Math. Soc. Janos Bolyai: Algebraic Theory of Semigroups 20(1976), 51-60.

7. V. Diekert; Some remarks on presentations by finite church Rosser Thue systems; Proceedings STACS 87, Lect. Notes Comp. Sci. 247(1987), 272-285.

8. M.J. Dunwoody; The accessibility of finitely presented groups; Inventiones Mathematicae 81(1985), 449-457.

9. R.H. Gilman; Computations with rational subsets of confluent groups; Proceedings of EUROSAM 84, Lect. Notes comp. Sci. 174(1984), 207-212.

10. R.H. Haring-Smith; Groups and simple languages; Trans. Amer, Math. Soc. 279(1983), 337-356.

11. R.C. Iyndon, P.E. Schupp; Combinatorial Group Theory, Springer-Verlag: Berlin, Heidelberg, New York, 1977.

12. K. Madlener, F. Otto; Commutativity in groups presented by finite Church-Rosser Thue systems, submitted for publication.

13. W. Magnus, A. Karrass, D. Solitar; Combinatorial Group Theory; 2nd revised ed., Dover Publ., New York, 1976.

14. D.E. Muller, P.E. Schupp; Groups, the theory of ends, and context-free languages; Journal Comp. System Sci. 26 (1983), 295-310.

15. M. Nivat; on some families of languages related to the Dyck languages; 2nd ACM Symp. on Theory of Comput. (1970), 221-225.

16. J.M. Autebert, L. Boasson, G. Senizergues; Groups and NTS languages; submitted for publication.

17. I.A. Wolf; Growth of finitely generated solvable groups and curvature of Riemannian manifolds; Journal Differential Geometry 2(1968), 421-446. 